\title{
Recent advances in holographic recording media for dynamic holographic display
}

Chao Ping Chen ${ }^{1}$, Yikai Su ${ }^{1}$ and Chul Gyu Jhun ${ }^{2 *}$

*Correspondence: cgjhun@hoseo.edu

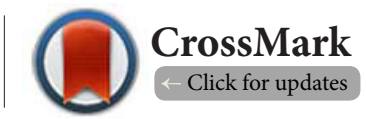

'National Engineering Lab for TFT-LCD Materials and Technologies, Department of Electronic Engineering, Shanghai Jiao Tong University, Shanghai 200240, China.

${ }^{2}$ School of Green Energy and Semiconductor Engineering, Hoseo University, Asan, Chungnam 336-795, Korea.

\begin{abstract}
An overview of the recent advances in the state-of-the-art holographic recoding media dedicated to the dynamic holography display is presented in this paper. Unlike the traditional holographic media, those materials enable the holograms to be updatable rather than permanent like before. Over the past few years, a number of material recipes for this use have been disclosed and can be mainly divided into four classesphotorefractive/photochromic polymers and photorefractive/photochromic liquid crystals. Focusing on five key material properties related to the display application, including diffraction efficiency, applied electric field, recording intensity, rise time and memory, the important findings as well as the underlying mechanisms are unraveled with in-depth discussion. Moreover, a comparison of the four classes of materials is provided, followed by a projection of the potential challenges.
\end{abstract}

Keywords: Holographic recording media, dynamic, holographic display, photorefractive, photochromic, doped liquid crystals

\section{Introduction}

Ever since the runaway success of science fiction blockbuster Avatar, a three-dimensional (3D) craze has been sweeping the whole world in recent years. According to one report announced by Allied Market Research, the global 3D technology market was valued at $\$ 46.0$ billion in 2013 , and is estimated to reach $\$ 175.1$ billion, by the end of 2020, growing at a compound annual growth rate (CAGR) of 21\% during 2013-2020 [1]. Technically, of all sorts of 3D displays, holography is considerd as an ultimate techiqnue for displaying 3D images [2]. Unlike the glasses-type 3D [3], it is inherently autostereoscopic as it allows the viewer to perceive the light as it would be scattered by the real object itself without any special eye wear. However, 67 years after its birth in 1947 [4]-almost 20 years older than liqud crystal display (LCD) [5]-holography is still a technology staying in the lab rather than going places in the commercial display markets. One reason, among others that could explain the above situation, is that in traditional holography, dichromated gelatin and silver halide are used as recording media [6], and those materials are incapable of updating holograms, thereby limiting their application merely to the optical storage of still pictures. In order to address this issue, computer-generated holography (CGH) [7] emerged as an alternative method, which bypasses the need of recording by digitally generating holograms. To live up to the standard video frame rate, a huge amount of information up to terabits/s needs to be processed [8]. In fact, this is impractical with the current computers. Another probelm bothering CGH lies in the dimension of the spatial light modulator (SLM), whereby the holograms are reconstructed. The most common SLM technologies adopted are liquid crystal on silicon (LCoS) [9] and micro-electro-mechanical systems (MEMS) [10]. Due to the process capability of semiconductors, both of them are quite limited in size, usually below 2 inches in diagonal [11]. Clearly, this falls far short of the expections for most 3D scenarios. Until 2008, a paper in Nature authored by Tay et al., disclosed an ingenious solution for the above problems [12]. Their basic concept is to refresh integral holographic images through an updatable photorefractive polymer. This would not only save the massive calculations, but be easily scalable in dimension. Inspired by this work, the search for an updatable recording medium with fast response and scalability becomes a hot topic. 
In this paper, the latest advances in the exploration and development of emerging holographic recording media are comprehensively surveyed. Per the types of the major components, materials of interest are categorized as photorefractive/photochromic polymers and photorefractive/ photochromic liquid crystals (LCs). Additionally, the prospects and challengenes toward the practical application are discussed, aiming to give deep insights into the material science and physics behind.

\section{Review \\ Holographic recording media Photorefractive polymers \\ Mechanism}

Photorefractive effect is a nonlinear optical effect that literally refers to a change in the refractive index of a material caused by light [13]. It can be understood with the following explanations [14,15]: (1) two beams of light intersect in the material to generate a pattern of dark and bright fringes; (2) electrons/holes in bright fringes can be photo-excited to be able to move freely throughout the material; (3) these electrons/holes move under the influence of an applied electric field until trapped in the dark fringes, along with holes/electrons left behind, creating a locally varying space charge field; (4) this varying space charge field in turn changes the refractive index of the local molecules. This effect is firstly observed in inorganic photorefractive crystals $[16,17]$, then organic photorefractive polymers emerge as an alternative for the advantages of large electro-optic response, ease of synthesis, and large area device fabrication. In general, the organic photorefractive polymer is a composite made from four components, including photoconductive polymer, chromophore, sensitizer, and plasticizer. Each of them plays an important role [15]. Photoconductive polymer, generally p-type conductor, offers electrons donation and holes transport. Its conductivity largely hinges on the hole mobility, which is a key parameter dictating the material's response time. Besides, it provides a structural support for other components. Chromophore is the nonlinear optical material, whose molecules are subject to an orientational or conformational change once exposed to the electric field of the irradiation, thereby altering the refractive index. Plasticizer is to lower the polymer's glass transition temperature $\left(\mathrm{T}_{\mathrm{o}}\right)$ for promoting the mobility as well as the reorientation of chromophore [18]. Sensitizer serves as an electron acceptor and improves the polymer's sensitivity to the visible and near-infrared wavelengths [19]. For the sake of showing a timeline of the recent advances, the materials exemplified in what follows are given in the time sequence.

\section{PATPD-CAAN:FDCST:ECZ}

This composite was reported by Tay et al., from Arizona University and Nitto Denko Technical Corporation in 2008 [12]. It consists of $50 \mathrm{wt} \%$ of polyacrylic tetraphenyldiaminobiphenyl
(PATPD): carbaldehyde aniline (CAAN) (photoconductive copolymer), $30 \mathrm{wt} \%$ of fluorinated dicyanostyrene (FDCST) (chromophore), and $20 \mathrm{wt} \%$ of carbazolylethylpropionate (CzEPA) (plasticizer). The copolymer adopted therein is to minimize the phase separation between the functional components usually seen in homopolymer photorefractive composites [20]. In their experiments, a 4×4-inch (active area), $100-\mu \mathrm{m}$ thick holographic film is demonstrated. The driving of the film is carryied out by the so-called "voltag kick-off technique"-starting with $9 \mathrm{kV}$ of voltage for recording and then shifting to $4 \mathrm{kV}$ after recording. As a result, the rise time is boosted dramatically from $20 \mathrm{~s}$ up to only $0.5 \mathrm{~s}$, but at the expense of diffraction efficiency (DE) down to $55 \%$, as shown in Figure 1. In our view, the most significant achievement of this work is the concept of using this material for a dynamic integral holography. By doing so, the computational load required for generating the display is reduced to the calculation of sets of straight two-dimensional (2D) images instead of calculating the sophisticated holograms from first principles [6]. For this reason, a figure of merit (FOM) is defined as the ratio of the memory (the time to self-erase) to rise time for the material design.

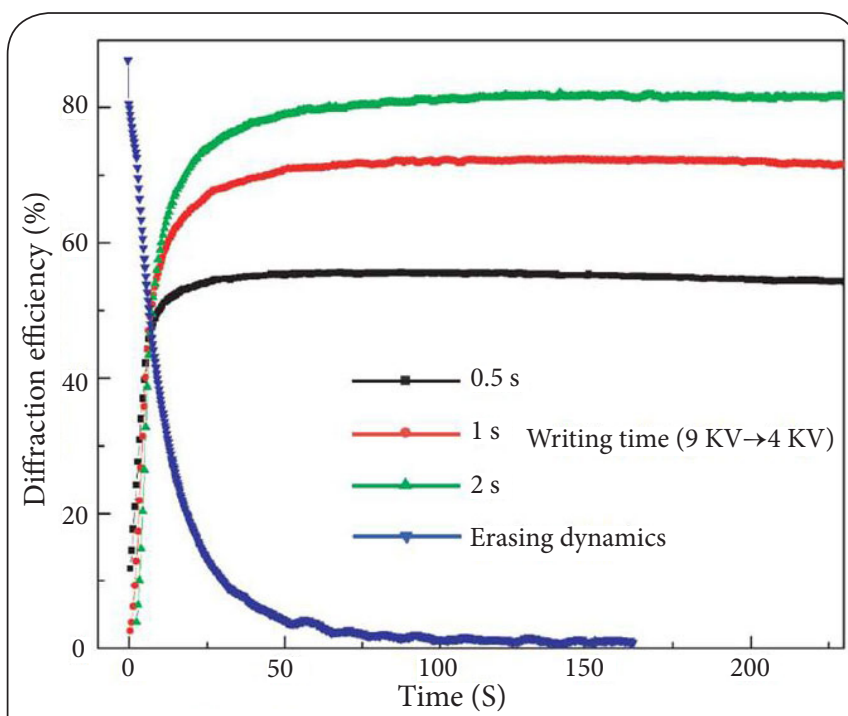

Figure 1. Recording dynamics of the photorefractive polymer (PATPD-CAAN:FDCST:ECZ ) using the voltage kick-off technique. The writing beams at $532 \mathrm{~nm}$ with $1 \mathrm{~W} / \mathrm{cm}^{2}$ irradiance are turned on at an applied voltage of $9 \mathrm{kV}$ for a few seconds (writing time), and then turned off. The voltage is then reduced to $4 \mathrm{kV}$ to ensure hologram persistence and high diffraction efficiency. The maximum diffraction efficiency achieved increases with increasing the writing time. Reproduced with permission. Copyright 2008, Nature [12].

\section{PVCz:7-DCST:CzEPA:TNF}

This composite was reported by Tsutsumi et al., from Kyoto Institute of Technology in 2012 [21]. It consists of $44 \mathrm{wt} \%$ of poly ( $N$-vinyl carbazole) PVCz (photoconductive polymer), 35 
wt $\%$ of 4-azacycloheptylbenzylidene-malonitrile (7-DCST) (chromophore), $20 \mathrm{wt} \%$ of carbazolylethylpropionate (CzEPA) (photoconductive plasticizer), and $1 \mathrm{wt} \%$ of 2,4,7-trinitro-9fluorenone (TNF) (sensitizer). Emphasis is placed on proportion of the plasticizer, which brings the $\mathrm{T}_{\mathrm{g}}$ down to the $-18.5^{\circ} \mathrm{C}$. The reduction in glass transition temperature facilitates the orientation of the dye molecules. It is also pointed out that higher molecular weight of PVCz would lead to both the higher $\mathrm{DE}$ and faster response time. The fabricated holographic film is $1.8 \mathrm{~cm}^{2}$ big and $60-100 \mu \mathrm{m}$ thick. Its DE and weighted average rise time are measured as $68 \%$ and $59 \mathrm{~ms}$, respectively, under an applied electric field of $45 \mathrm{~V} / \mu \mathrm{m}$ and a writing intensity of $1.5 \mathrm{~W} / \mathrm{cm}^{2}$. Both a real object (coin) hologram and a twodimensional (2D) image rendered on a SLM are demonstrated.

\section{PDAA:7-DCST:PCBM:BBP}

This composite was reported by Giang et al., from Kyoto Institute of Technology in 2012 [22]. It consists of $55 \mathrm{wt} \%$ of poly (4-(diphenylamino)benzyl acrylate) (PDAA) (photoconductive polymer), $40 \mathrm{wt} \%$ of 7-DCST (chromophore), $4 \mathrm{wt} \%$ of benzyl $\mathrm{n}$-butyl phthalate (BBP) (plasticizer), and $1 \mathrm{wt} \%$ of phenyl-C61butyric acid methyl ester (PCBM) (sensitizer). Highlighted is the triphenylamine moiety of the photoconductive polymer, which has a fast hole mobility of $10^{-4}-10^{-3} \mathrm{~cm}^{2} / \mathrm{V} \cdot \mathrm{s}$. This will result in a fast response time, as the hole mobility governs the speed of the space charge field formation. In addition, the plasticizer BBP is assisted with the acrylate in the PDAA to further reduce $T_{g}$ as well as viscosity. It is also mentioned that a low viscosity is favorable for making the large-size holographic film. In their experiments, the biggest size of the film is 3.1 inches in diagonal. The measured DE and rise time are $80 \%$ and $267 \mathrm{~ms}$, respectively, under an applied electric field of $45 \mathrm{~V} / \mu \mathrm{m}$ and a writing intensity of $172 \mathrm{~mW} / \mathrm{cm}^{2}$.

\section{Photochromic polymers Mechanism}

Photochromism is defined as a reversible transformation of a chemical species between two forms having different absorption spectra [23]. In chemistry, isomerization is typical of a photochromism. During the isomerization, not only the absorption spectra but also various physicochemical properties change, such as the refractive index, dielectric constant, oxidation/reduction potential, and geometrical structure. For photochromic polymers, there are two isomers-molecules with the same atoms but different structures-switchable depending on the light or the heat. Take azobenzene, known for its trans-cis isomerization [24], as an instance. The two isomers can be switched with particular wavelengths of light, of which, ultraviolet (UV) light is for trans-to-cis conversion, and blue light for cis-to-trans. Since the whole process only involves with light, photochromic polymers can work without any electric field. To act as updatable recording media, the photochromic polymers are supposed to possess fast response, thermal stability of both isomers, resistance to fatigue during cyclic write-and-erase, and high sensitivity [14].

Azobenzene side-chained copolymer:LC(E63)

In 2012, Wu et al., from New Span Opto-Technology and Univ. of Miami introduced a polymer-liquid crystal (LC) colloid-like material $[25,26]$. It has a quite unique configuration in that the azobenzene side-chained methacrylate-aliphatic copolymers are dispersed within the fluid LCs. Upon the exposure, the isomerization of azobenzene molecules on the side chain will deform the polymer's main chain, causing a phenomenon called the photomobile effect [27]. The LCs surrounding the polymer chains are therefore redistributed to change the refractive index. The most impressive attribute about this material is its excellent memory that could last for months at room temperature and in ambient lighting conditions (estimated at least one year in a dark condition). This is due to the bulky size of macromolecules like polymers, who require a longer time for a mechanical recovery. And this is also why its rise time is nearly $50 \mathrm{~s}$.

\section{NACzE:PMMA}

In 2012, Tsutsumi et al., from Kyoto Institute of Technology and Chitose Institute of Science and Technology introduced a monolithic organic compound of 3-[(4-nitrophenyl)azo]9Hcarbazole-9-ethanol (NACzE) (30 wt\%) doped transparent polymethylmethacrylate (PMMA) [28]. The nitrophenyl azo unit in the NACzE accounts for the mechanism as isomerization, which is conducted in an all-optical way, saving thousands of volts of driving voltage necessary for the photorefractive polymers. The fabricated holographic film is $7.5 \times 5 \mathrm{~cm}^{2}$ big and $50 \mu \mathrm{m}$ thick. Its opitcal gain is $451 \mathrm{~cm}^{-1}$ measured using two-beam coupling method [15], and DE is $40 \%$ measured at $632.8 \mathrm{~nm}$ using the four-wave mixing [15]. Although the authors title it as "real-time 3D holographic display" with a rise time greater than $30 \mathrm{~s}$, it might be more suitabe for the application as signage where infrequent reresh rate is needed.

\section{PC-bridged imidazole dimer}

In 2012, Ishii et al., from Aoyama Gakuin University and Japan Science and Technology Agency introduced a photochromic [2.2]paracyclophane (PC)-bridged imidazole dimer [29]. This dimer, upon the exposure to UV light, exhibits coloration from colorless to blue, with its $\mathrm{C}-\mathrm{N}$ bond between the two imidazole rings being homolytically cleaved to give a pair of imidazolyl radicals. A $170-\mu \mathrm{m}$ thick holographic film is prepared by a simple solution-casting method from the benzene solution of the mixture of the photochromic molecule, poly (ethyl acrylate), and poly (phenoxyethyl acrylate) at a ratio of $1: 1: 2 \mathrm{wt} \%$. Its dynamic property is demonstrated by tracing the movement of a patterned mask with instantly diffracted images at a time interval of $0.5 \mathrm{~s}$. Besides, after reaching the maximum DE for a rise time of $300 \mathrm{~ms}$, the images can be held up for 100 ms, as depicted in Figure 2. The limitation about this material lies in the $\mathrm{DE}$, which is below $0.1 \%$. The authors 


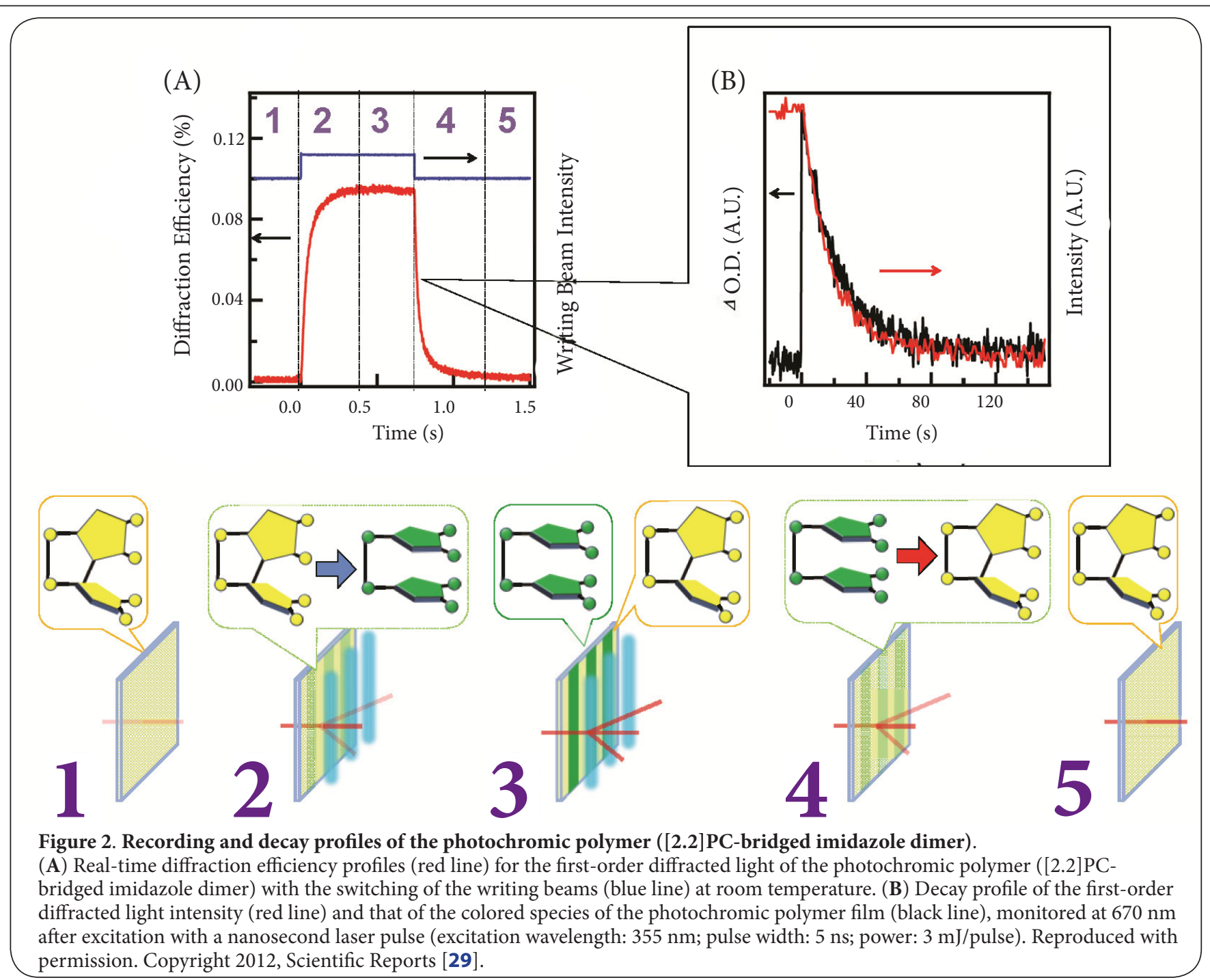

mainly attribute this low $D E$ to the strong absorption of the molecules in the colored form, thinning the effective grating thickness to be the Raman-Nath type [30].

\section{Photorefractive liquid crystals Mechanism}

In principle, LCs that are free of impurities are good insulators and charges must be provoked by some means before photorefractive effect can occur [31]. The mostly adopted ways are done by doping LCs with semiconducting particles [32-35] or coating the substrate with semiconducting layers $[36,37]$. The semiconducting particles/layers are capable of producing electron-hole pairs, namely excitons, after absorbing photons with energies exceeding the bandgap of the material. Upon the application of an electric field across the LC cell, the electron-hole pairs are separated so as to form the space charge field. To evaluate the performance of photorefractive LCs, one can consider the mobility of charge carriers in LCs and the quantum efficiency of semiconducting nanoparticles/layers - the ratio of the number of converted electrons to the number of incident photons. Compared to the four components required for photorefractive polymers, the composition of photorefractive LCs is apparently simpler. As an aside, the LC-based holographic devices can be easily fabricated at sufficiently large sizes, thanks to the today's mature LCD technologies.

\section{$C_{60}$-doped nematic LC (5CB)}

In 2012, Lian et al., from Harbin Institute of Technology proposed a $\mathrm{C}_{60}$-doped 4,4'-npentylcyanobiphenyl (5CB) LC [37]. In addition to doping, there are two semiconducting $\mathrm{ZnSe}$ layers enclosing the LC. By doing so, the photorefractive effect is remarkably intensified as the gain coefficient is up to 350 $\mathrm{cm}^{-1}$. If replaced with bare glass substrates, the same device will see no appreciable nonlinear optical effect, even at a voltage of $1000 \mathrm{~V}$. Since the space charge filed is induced in parallel with the surface, the LC is aligned homeotropically so as to maximize the rotation angle. Using the four-wave 
Chen et al. Journal of Optics and Photonics 2014,

mixing method, its rise time and DE are measured as $20 \mathrm{~ms}$ and $8 \%$, respectively.

\section{T-2MB:TNF-doped smectic LC}

In 2013, Sasaki et al., from Tokyo University of Science proposed a ferroelectric LC doped with $10 \mathrm{wt} \%$ of $3 \mathrm{~T}-2 \mathrm{MB}$ (chiral dopant) and $0.1 \mathrm{wt} \%$ of TNF [38]. The ferroelectric LC is often characterized by a spontaneous electric polarization that can be reversed by the application of an external or internal electric field. Reinforced by this spontaneous polarization, the space charge field could rotate LC molecules more easily and faster. Hence, the applied electric field is only $1 \mathrm{~V} / \mu \mathrm{m}$ and the rise time is $8 \mathrm{~ms}$. As shown in Figure 3 , it has also been found out

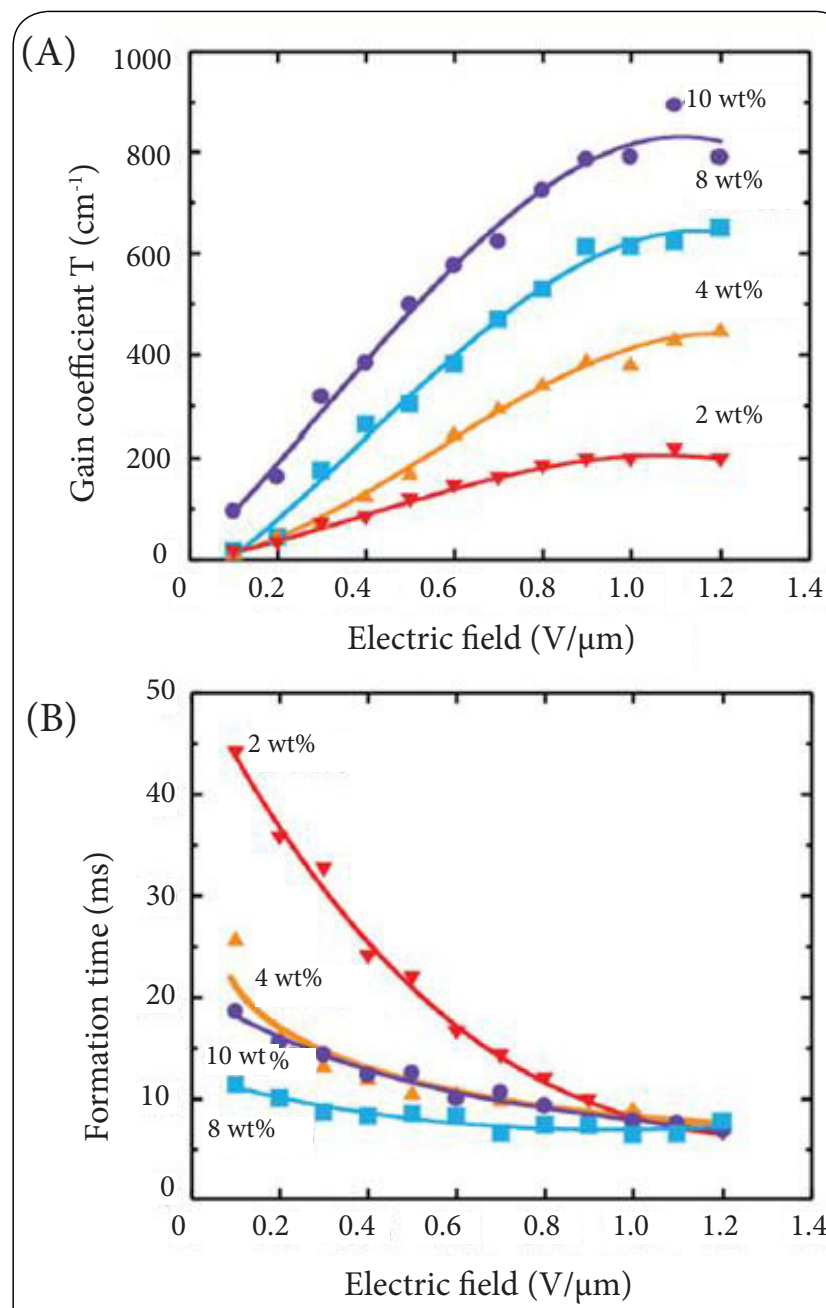

Figure 3. Electrical field dependence of gain coefficients and refractive index grating formation time of 3T-2MB:TNFdoped smectic LC.

(A) Electric field dependence of gain coefficients of mixtures of 3T-2MB:TNF-Doped Sematic LC measured at $30^{\circ} \mathrm{C}$. 3T$2 \mathrm{MB}$ concentration was in the range $2-10 \mathrm{wt} \%$. (B) Refractive index grating formation time (response time) of mixtures of 3T-2MB:TNF-doped smectic LC measured at $30^{\circ} \mathrm{C}$. 3T-2MB concentration was in the range $2-10 \mathrm{wt} \%$. Reproduced with permission. Copyright 2013, Applied Physics Letters [38]. that a higher concentration of chiral dopant yields a better performance owing to the photoconductivity based on the hopping mechanism [39].

\section{ZnS/InP-doped nematic LC(5CB)}

In 2014, Li et al., from Shanghai Jiao Tong Univeristy proposed a mixture of a nematic LC (5CB) doped with $0.05 \mathrm{wt} \%$ of quantum dots (Zns/lnP) [40]. The quantum dots used therein are characterized by a core-shell structure, which is quite effective in enhancing the quantum efficiency by passivating the surface trap states [41]. This enhanced quantum efficiency is responsible for its DE about $20 \%$ being higher than the previous two. It has also indicated that the shorter the grating pitch becomes, the faster the rise time is. This is legitimate considering that the charge carries will drift or diffuse at a shorter distance. At an applied voltage of $17 \mathrm{~V}$, the fastest rise time measured is $6.4 \mathrm{~ms}$, while the memory time is $140 \mathrm{~ms}$, as shown in Figure 4.

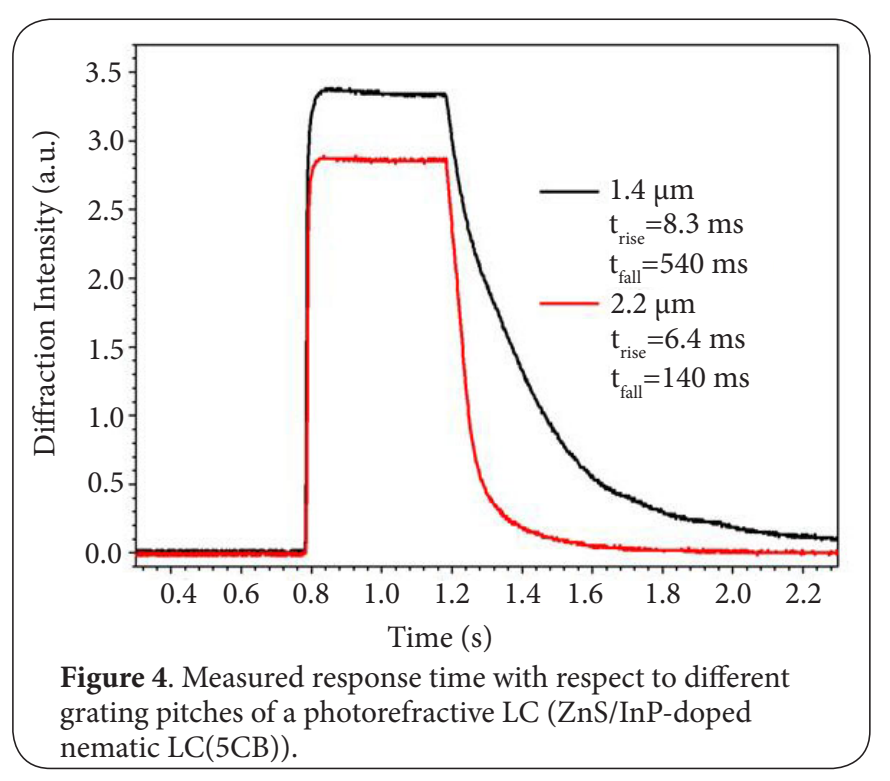

\section{Photochromic liquid crystals Mechanism}

Photochromic liquid crystals, especially those doped with azo dyes, occupy an important niche in nonlinear optics on account of their extraordinarily large optical nonlinearities [42]. It was once reported by Lucchetti et al [43]. that the nonlinearity of methyl-red doped nematic LCs measured was greater than $10^{3} \mathrm{~cm}^{2} / \mathrm{W}$. For holographic display, a large nonlinearity would translate into a lower threshold of laser power. Quite different from photochromic polymers, whose nonlinearity is caused by a conformational change, $\mathrm{LCs}$ rotate their molecular axes without changing in shape $[\mathbf{4 4 , 4 5}]$. This in part explains the large nonlinearity they have. To act as updatable recording media, it is preferable for photochromic LCs to have a large birefringence, a low viscosity, and a high clearing point [42]. 
Chen et al. Journal of Optics and Photonics 2014,

http://www.hoajonline.com/journals/pdf/2057-1569-1-1.pdf

doi: $10.7243 / 2057-1569-1-1$

\section{DR1-doped nematic LC (5CB)}

In 2014, Li et al., from Shanghai Jiao Tong Univeristy proposed a mixture of a nematic LC (5CB) doped with $4 \mathrm{wt} \%$ of an azo dye (disperse red 1, DR1) $[46,47]$. As shown in Figure 5, it exhibits a rise time as fast as $1.8 \mathrm{~ms}$, which is unparalleled by others mentioned so far. The memory, on the other hand, is relatively short being only $4.8 \mathrm{~ms}$. Because of the anistropic nature of both $L C$ and dye, the response time as well as $D E$ to a degree depends on the polarization direction of the recording beams. When the polarization coincides with the LC director, same as the absorption axis of the dye, the nonlinear optical effect maximizes. Another important finding is the ceiling of the recording intensity, over which the as-recorded gratings becomes vanished. This arises from the thermal effect [44] that would heat LC to its clearing point, at which LC turns out to be isotropic. For its quick response, the authors realize a video-rate holographic display with a refresh rate

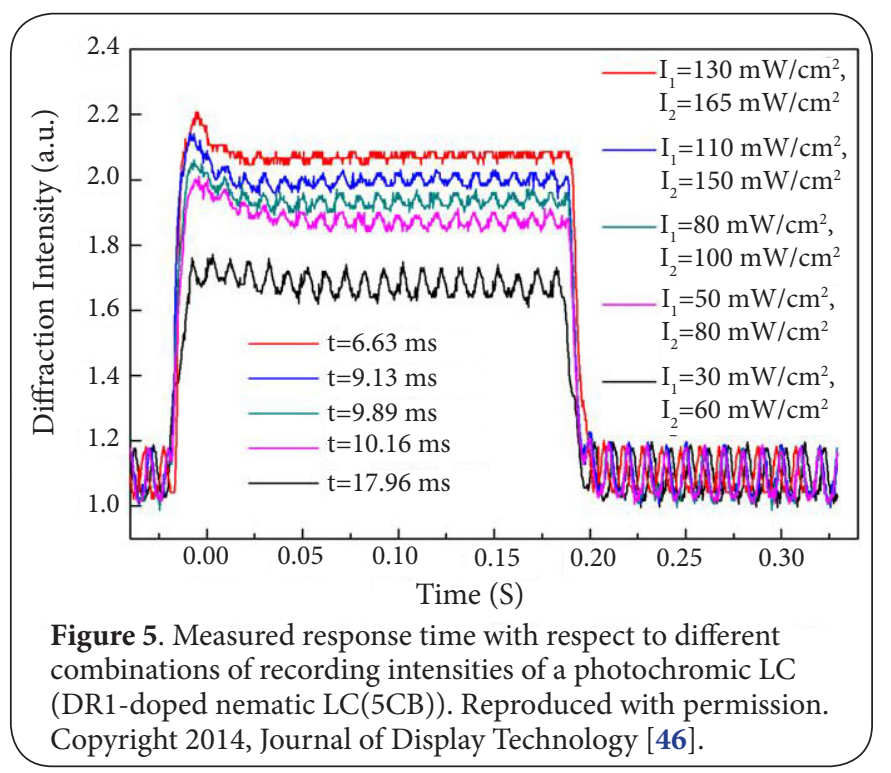

of $25 \mathrm{~Hz}$. In addition, the multiplexing capability for a color display has also been verified [47].

\section{Conclusions}

This paper reviews the current state-of-the-art of the holographic recording media specialized for the dynamic holographic display, as with those materials, holograms can be rewritable and scalable to a large size. Of various nonlinear optical materials, photorefractive/photochromic polymers, photorefractive/photochromic LCs are identified as the four most promising candidates for the above purpose. To evaluate the material properties essential for the display application, typical performance indicators, including $D E$, applied electric field, recording intensity, rise time, and memory, are listed in Table 1. By comparison, photorefractive polymers stand out in getting high DE up to $90 \%$. This would translate into brighter holographic images for the same power input. Photochromic polymers on average have edges over others in terms of the null electric field, relatively low recording intensities, and excellent memory to self-erase holograms. Photorefractive LCs are mediocre with all performance being ranked in middle. Last but not least, photochromic LCs are considered as an ideal choice for the video-rate display applications, since the case using the azo-dye doped liquid crystals can have a rise time to be as fast as $1.8 \mathrm{~ms}$. Regarding the future holographic recording media, it is expected that the photorefractive/ photochromic polymers and photorefractive/photochromic LCs will continue to be the most active materials in this field. From the perspective of display for practical uses, more material parameters need to be taken into account, such as sensitivity, resolution, viewing angle, shelf life etc. While these recent years have seen considerable progress in both physics and material science, there is still a long way for holography to go in the next few decades.

\section{Competing interests}

The authors declare that they have no competing interests.

Table 1. Comparison of diffraction efficiency, applied electric field, recording intensity, rise time and memory for various photorefractive/photochromic polymers and photorefractive/photochromic liquid crystals.

\begin{tabular}{|c|c|c|c|c|c|c|c|}
\hline Materials & Classes & $\begin{array}{l}\text { Diffraction } \\
\text { efficiency (\%) }\end{array}$ & $\begin{array}{l}\text { Applied electric } \\
\text { field }(\mathrm{V} / \mu \mathrm{m})\end{array}$ & $\begin{array}{l}\text { Recording intensity } \\
\left(\mathrm{mW} / \mathrm{cm}^{2}\right)\end{array}$ & Rise time & Memory & Year \\
\hline PATPD-CAAN:FDCST:ECZ & $\mathrm{PR}^{\mathrm{a}}$ polymers & 55 & 90 & 1000 & $500 \mathrm{~ms}$ & hours & 2008 \\
\hline PVCz:7-DCST:CzEPA:TNF & PR polymers & 68 & 45 & 1500 & $59 \mathrm{~ms}$ & unknown & 2012 \\
\hline PDAA:7-DCST:PCBM:BBP & PR polymers & $>80$ & 40 & 172 & $267 \mathrm{~ms}$ & unknown & 2013 \\
\hline $\begin{array}{l}\text { Azobenzene side-chained } \\
\text { methacrylate/LC(E63) }\end{array}$ & $\mathrm{PC}^{\mathrm{b}}$ polymers & $>10$ & 0 & 159 & $50 \mathrm{~s}$ & months & 2012 \\
\hline NACzE/PMMA & PC polymers & 40 & 0 & 16.6 & $>30 \mathrm{~s}$ & hours & 2012 \\
\hline PC-bridged imidazole dimer & PC polymers & $<0.1$ & 0 & 1200 & $300 \mathrm{~ms}$ & $100 \mathrm{~ms}$ & 2012 \\
\hline $\mathrm{C}_{60}$-doped nematic LC(5CB) & PR LCs & 8 & 0.23 & 300 & $20 \mathrm{~ms}$ & $\sim 25 \mathrm{~ms}$ & 2012 \\
\hline 3T-2MB:TNF-doped smectic LC & PR LCs & $2 \sim 3$ & 1 & 159 & $8 \mathrm{~ms}$ & unknown & 2013 \\
\hline ZnS/InP-doped nematic LC(5CB) & PR LCs & 20 & 1.4 & 260 & $6.4 \mathrm{~ms}$ & $140 \mathrm{~ms}$ & 2014 \\
\hline DR1-doped nematic LC(5CB) & PC LCs & 0.6 & 0 & 295 & $1.8 \mathrm{~ms}$ & $4.8 \mathrm{~ms}$ & 2014 \\
\hline
\end{tabular}

aPR: Photorefractive; ${ }^{\mathrm{b}} \mathrm{PC}$ : Photochromic 
Chen et al. Journal of Optics and Photonics 2014,

\section{Acknowledgement}

This work is sponsored by 973 Program (2013CB328804), National Natural Science Foundation of China (61307028), and Science \& Technology Commission of Shanghai Municipality (13ZR1420000). Publication history

EIC: Vincenzo Eramo, University of Rome, Italy.

Received: 20-Jun-2014 Final Revised: 01-Oct-2014

Accepted: 08-Oct-2014 Published: 22-Oct-2014

\section{References}

1. Allied Market Research. Three-Dimensional Technology Market Global Forecast (2012-2020). I Website

2. C. W. Christenson, P.-A. Blanche, S. Tay, R. Voorakaranam, T. Gu, W. Lin, P. Wang, M. Yamamoto, J. Thomas, R. A. Norwood and N. Peyghambarian. Materials for an Updatable Holographic 3D Display. J. Disp. Technol. 2010; 6:510. | Article

3. J. Geng. Three-dimensional display technologies. Adv. Opt. Photon. 2013; 5:456. | Article |

4. D. Gabor. The British Thomson-Houston Co. Ltd. GB 685286A. 1947.

5. Chen CP, Lee JH, Yoon TH and Kim JC. Monoview/dual-view switchable liquid crystal display. Opt Lett. 2009; 34:2222-4. I Article I PubMed

6. V. Toal. Introduction to Holography. CRC Press, Boca Raton, FL, US. 2011.

7. A. Georgiou, J. Christmas, N. Collings, J. Moore and W. A. Crossland. Aspects of hologram calculation for video frames. J. Opt. A: Pure Appl. Opt. 2008; 10:035302. | Article

8. L. Gharai and C. Perkins. Holographic and 3D Teleconferencing and Visualization: Implications for Terabit Networked Applications. $25^{\text {th }}$ IEEE Int. Conf. Comput. Commun., Barcelona, Spain. 2006. | Article

9. A. Michalkiewicz, M. Kujawinska, T. Kozacki, X. Wang and P. J. Bos. Holographic three-dimensional displays with liquid crystal on silicon spatial light modulator. Proc. SPIE. 2004; 5531:85. | Article

10. Srinivas A. Tadigadapa, and Nader Najafi. Developments in Microelectromechanical Systems (MEMS): A Manufacturing Perspective. J. Manuf. Sci. Eng. 2003; 125:816. | Article

11. D. Armitage, I. Underwood and S.-T. Wu, Introduction to Microdisplays. Wiley, Chichester, West Sussex, UK. 2006.

12. Tay S, Blanche PA, Voorakaranam R, Tunc AV, Lin W, Rokutanda S, Gu T, Flores D, Wang P, Li G, St Hilaire P, Thomas J, Norwood RA, Yamamoto $\mathrm{M}$ and Peyghambarian N. An updatable holographic three-dimensional display. Nature. 2008; 451:694-8. | Article | PubMed

13. G. C. Valley, M. B. Klein, R. A. Mullen, D. Rytz and B. Wechsler. Photorefractive Materials. Ann. Rev. Mater. Sci. 1988; 18:165.

14. A. B. Samui. Holographic Recording Medium. Recent Patents on Materials Science. 2008; 1:74. I Article

15. B. Lynn, P.-A. Blanche and N. Peyghambarian. Photorefractive Polymers for Holography. J. Polym. Sci., Part B: Polym. Phys. 2014; 52:193. | Article

16. A. Ashkin, G. D. Boyd, J. M. Dziedzic, R. G. Smith, A. A. Ballman, J. J. Levinstein and K. Nassau. Optically-induced refractive index inhomogeneities in $\mathrm{LiNbO}_{3}$ and $\mathrm{LiTaO}_{3}$. Appl. Phys. Lett. 1966; 9:72. | Article

17. A. Twarowski. Geminate recombination in photorefractive crystals. J. Appl. Phys. 1989; 65:2833. | Article

18. Blanche PA, Bablumian A, Voorakaranam R, Christenson C, Lin W, Gu T, Flores D, Wang P, Hsieh WY, Kathaperumal M, Rachwal B, Siddiqui O, Thomas J, Norwood RA, Yamamoto M and Peyghambarian N. Holographic three-dimensional telepresence using large-area photorefractive polymer. Nature. 2010; 468:80-3. | Article I PubMed

19. S. Kober, F. Gallego-Gomez, M. Salvador, F. B. Kooistra, J. C. Hummelen, K. Aleman, S. Mansurova and K. Meerholz. Influence of the sensitizer reduction potential on the sensitivity of photorefractive polymer composites. J. Mater. Chem. 2010; 20:6170. | Article

20. G. C. Eastmond and D. G. Phillips. Macroscopic phase separation in multicomponent polymer homopolymer blends: general considerations based on studies of AB-crosslinked polymers. Polymer. 1979; 20:1501. I Article

21. N. Tsutsumi, K. Kinashi, A. Nonomura and W. Sakai. Quickly Updatable Hologram Images Using Poly(N-vinyl Carbazole) (PVCz) Photorefractive Polymer Composite. Materials. 2012; 5:1477. | Article

22. H. N. Giang, K. Kinashi, W. Sakai and N. Tsutsumi. Photorefractive response and real-time holographic application of a poly(4(diphenylamino)benzyl acrylate)-based composite. Polym. J. 2014; 46:59. I Article

23. Irie M. Photochromism: Memories and Switches-Introduction. Chem Rev. 2000; 100:1683-1684. | Article | PubMed

24. P. Wu, D. V. G. L. N. Rao, B. R. Kimball, M. Nakashima and B. S. DeCristofano. Nonvolatile grating in an azobenzene polymer with optimized molecular reorientation. Appl. Phys. Lett. 2001; 78:1189. | Article

25. Wu P, Sun SQ, Baig S and Wang MR. Nanoscale optical reinforcement for enhanced reversible holography. Opt Express. 2012; 20:3091-7. I Article I PubMed

26. Wu P, Sun SQ, Baig S and Wang MR. Enhanced non-volatile and updatable holography using a polymer composite system. Opt Express. 2012; 20:6052-7. | Article | PubMed

27. Yamada M, Kondo M, Mamiya J, Yu Y, Kinoshita M, Barrett CJ and Ikeda T. Photomobile polymer materials: towards light-driven plastic motors. Angew Chem Int Ed Engl. 2008; 47:4986-8. | Article I PubMed

28. N. Tsutsumi, K. Kinashi, W. Sakai, J. Nishide and Y. Kawabe. Real-time three-dimensional holographic display using a monolithic organic compound dispersed film. Opt. Mater. Express. 2012; 2:1003. I Article

29. Ishii N, Kato T and Abe J. A real-time dynamic holographic material using a fast photochromic molecule. Sci Rep. 2012; 2:819. | $\underline{\text { Article | PubMed }}$ Abstract | PubMed Full Text

30. Moharam MG and Young L. Criterion for Bragg and Raman-Nath diffraction regimes. Appl Opt. 1978; 17:1757-9. I Article I PubMed

31. Liu CY and Bard AJ. Pressure-induced insulator conductor transition in a photoconducting organic liquid-crystal film. Nature. 2002; 418:162-4. | Article I PubMed

32. A. Anczykowska, S. Bartkiewicz, M. Nyk and J. Mysliwiec. Study of semiconductor quantum dots influence on photorefractivity of liquid crystals. Appl. Phys. Lett. 2012; 101:101107. | Article

33. I. C. Khoo, J. Ding, Y. Zhang, K. Chen and A. Diaz. Supra-nonlinear photorefractive response of single-walled carbon nanotube- and $\mathrm{C}_{60}{ }^{-}$ doped nematic liquid crystal. Appl. Phys. Lett. 2003; 82:3587. I Article

34. S. E. San and O. Koysal. An outstanding holographic composite employing methyl red and fullerene $\mathbf{C 6 0}$ under the same liquid crystal structure. Displays. 2003; 24:209. | Article

35. S. Ghosh and G. O. Carlisle. Carbon nanotube enhanced diffraction efficiency in dye-doped liquid crystal. J. of Mater. Sci.: Mater. Electron. 2005; 16:753. | Article

36. Sahraoui B, Anczykowska A, Bartkiewicz S and Mysliwiec J. Optimization of liquid crystal structures for real time holography applications. Opt Express. 2011; 19:24454-9. | Article | PubMed

37. Lian $\mathrm{C}$, Zhao $\mathrm{H}$, Pei $\mathrm{Y}$, Sun $\mathrm{X}$ and Zhang J. Fast response beam coupling in liquid crystal cells sandwiched between ZnSe substrates. Opt Express. 2012; 20:15843-52. | Article | PubMed

38. T. Sasaki, M. Ikegami, T. Abe, D. Miyazaki, S. Kajikawa and Y. Naka. Realtime dynamic hologram in photorefractive ferroelectric liquid crystal. Appl. Phys. Lett. 2013; 102:063306. | Article

39. R. K. Hovsepyan, N. R. Aghamalyan and S. I. Petrosyan. Hopping and Drift Mechanisms of Photoconductivity in ZnO:Li Films. J. Contemp. Phys. 2009; 44:29. I Article

40. X. Li, C. P. Chen, Y. Li, W. Hu, H. Li, X. Jiang, N. Rong, Y. Yuan, J. Lu and Y. Su. Real-Time Holographic Display Using Quantum Dot Doped Liquid Crystal. SID Symposium Digest. 2014; 45:736. | Article

41. Reiss P, Protiere M and Li L. Core/Shell semiconductor nanocrystals. Small. 2009; 5:154-68. | Article | PubMed 
Chen et al. Journal of Optics and Photonics 2014,

http://www.hoajonline.com/journals/pdf/2057-1569-1-1.pdf

42. I. C. Khoo. Nonlinear optics of liquid crystalline materials. Phys. Rep. 2009; 471:221. | Article

43. Lucchetti L, Gentili M and Simoni F. Colossal optical nonlinearity induced by a low frequency external electric field in dye-doped liquid crystals. Opt Express. 2006; 14:2236-41. | Article | PubMed

44. Fuh AY, Lin HC, Mo TS and Chen CH. Nonlinear optical property of azodye doped liquid crystals determined by biphotonic Z-scan technique. Opt Express. 2005; 13:10634-41. | Article | PubMed

45. C. P. Chen, K. H. Kim, T.-H. Yoon and J. C .Kim. A Viewing Angle Switching Panel Using Guest-Host Liquid Crystal. Jpn. J. Appl. Phys. 2009; 48:062401. | Article

46. X. Li, C. P. Chen, H. Gao, Z. He, Y. Xiong, H. Li, W. Hu, Z. Ye, G. He, J. Lu and Y. Su. Video-Rate Holographic Display Using Azo-Dye-Doped Liquid Crystal. J. Disp. Technol. 2014; 10:438. I Article

47. X. Li, C. P. Chen, Y. Li, X. Jiang, H. Li, W. Hu, G. He, J. Lu and Y. Su. Color holographic display based on azo-dye-doped liquid crystal. Chin. Opt Lett. 2014; 12:060003. | Article

\section{Citation:}

Chen CP, Su Y and Jhun CG. Recent advances in holographic recording media for dynamic holographic display. J Opt Photonics. 2014; 1:1. http://dx.doi.org/10.7243/2057-1569-1-1 